\title{
Frequency and Voltage Control of Island System using Power Hardware In the Loop
}

\author{
Solomon Oyegoke \\ Faculty of Engineering and Science University \\ of Greenwich, Medway, Kent, United Kingdom \\ O.Solomon@greenwich.ac.uk
}

\author{
Marios Maniatopoulos \\ School of Electrical and Computer \\ Engineering, National Technical \\ University of Athens, Athens, Greece \\ mmaniato@mail.ntua.gr \\ Yehdego Habtay \\ Faculty of Engineering and Science \\ University of Greenwich, Medway, \\ Kent, United Kingdom \\ Y.T.Habtay@greenwich.ac.uk
}

\author{
Simeon Keates \\ School of Engineering and the Built \\ Environment, Edinburgh Napier University, \\ Edinburgh,United Kingdom \\ S.Keates@napier.ac.uk
}

\begin{abstract}
Power generation via Distributed Generation (DG) plants coupled with inverters can participate in Frequency and Voltage stabilization of the microgrid which is important to grid operators.
\end{abstract}

This paper explores the realization of improved regulation of frequency and voltages in a low voltage microgrid using droopcontrolled power converters. The test bed consists of a Power Hardware in the Loop (PHIL) setup, which includes a gridforming battery inverter, local loads, and DG plants.

In the PHIL setup, the grid-forming battery inverter was responsible for setting the network voltage, which served the loads initially before the distributed energy resources were energized. Additional loads were then switched on triggering a frequency drop in the network. Changes in the load requirement and performance of the DGs were examined through the PHIL setup with the energy storage system further supporting as require. The results showed notable improvement in the overall system frequency and voltage regulation.

Index Terms-DERs, Ancillary Services, PHIL, Microgrid, Battery Energy Storage System, Solar PV, Wind, Power Quality.

\section{INTRODUCTION}

$\mathrm{D}$ istributed Energy Resources (DERs) as tools for power management are being increasingly explored for their potential to support existing conventional power generators. Most electrical grids are expected to become smarter in the near future $[1,2]$. The integration of renewable energy sources into power systems yields economic, technical and environmental benefits for the distribution system and the consumers [2]. DERs can also improve power quality because they feed loads locally and they can participate in frequency and voltage regulation. On the other hand, renewable energy sources are intermittent sources because of their dependency on external factors such as weather in generating power [2].

In realizing a smarter grid, these Distributed Generation (DG) plants coupled with local loads and potentially storage have formed the basis of a microgrid. Some of the microgrid functionalities include frequency and voltage stability, active and reactive power flow control, active-power filter abilities, energy storage management and black start operation which is used for restoring power in the event of a total or partial shutdown $[2,3]$. When considering an island microgrid which may be regarded as an isolated part of a main electric grid, the addition of renewable energy sources and the security of supply becomes a major point that must be addressed [4]. DG plants become responsible for sharing their load power demand, while maintaining the system frequency and voltage.

Energy storage systems (ESS) have the capability to provide viable reductions in the fuel consumption of diesel generators in island grids with medium to high penetration of DERs [5]; the IEEE-1547.4-2011 standard provides guidance on the design, operation and integration of Distributed Resource Island system into an existing Electrical Power System [6]. Frequency regulation can be achieved via Demand Response; the aggregation of Demand Response tools can be implemented to absorb excess power generated from renewable energy sources when demand is at minimum level [7].

In setting up and testing the island grid, the Power Hardware in the Loop (PHIL) concept is applied in this research. PHIL is described as a unique approach to testing, because different power systems can be scaled down. It can be tested under realistic conditions with a Hardware Under Test (HUT) device connected to a real-time simulator [8]. The scalability of equipment therefore reduces overall cost and space required for hardware equipment. By utilizing PHIL simulation, experiment and in-depth study can be carried out in controlled lab environment on power conversion, faults, load management, power flow analysis etc. $[10,13]$.

The Controller Hardware in the Loop (CHIL) simulation is another type of HIL simulation which is used for low-level digital or analogue testing [9]. Both CHIL and PHIL been discussed extensively and used in different power applications [8-16, 26-29].

\section{ANCILlary Services IN MicrogridS}

The microgrid can be described or seen as a cluster of DG plants, storage systems and loads that are interconnected and hierarchically controlled, with the capability to function either 
in grid-connected mode or island system [17]. For this case study we focus on the non-interconnected islands (NIIs) or island grid scenario. In the microgrid architecture the three levels of operation are Primary, Secondary and Tertiary Control [18, 19, 31-33]. The level of control depends on the operating state of the microgrid.

\section{A. Microgrid Architecture and Hierarchy}

The Primary control has a fast decision time and it describes the mode of operation where the DG plants are responsible for load sharing; in this mode, the local loads are powered via the DG plants. At the point where there is a mismatch between load requirement and amount of power generated in the microgrid, there may be a need to employ a voltage or frequency restoration technique to create a balanced network and this is known as secondary control [18]. Both Primary and Secondary control in the microgrid architecture can be employed for gridtied or island microgrid [18-19].

The tertiary control is the link between the microgrid and main grid; it ensures economic optimization, based on energy cost and electricity market [20]. It makes decisions to improve the overall performances of microgrid through system state information gathered via communication infrastructure and has an extended decision time step compared to that of the primary or secondary control [19]. In realising the complete operating objectives, the information that flows from the primary level to the tertiary level is the system state information and likewise, the decision signals flow from tertiary to the primary level. The tertiary control coordinates the ancillary service exchanged between the main-grid and microgrid [20].

The ability to run the microgrid as an island network means power will only be supplied by local generators to loads and there is no requirement for voltage synchronization, as would be the case were it to be connected to the main grid. In establishing an island microgrid, it is therefore important to examine demand from loads and how the system is best optimized to meet that demand.

In an island mode or off-grid network, voltage regulation and contribution of real and reactive power from the DG plants must therefore be addressed. Various control modes can be applied to the grid network, such as centralized control, but require a good data transfer and robust communication design [30]. Centralized control approaches may be suitable for an island grid where the DERs are installed close to each other. In instances where the DG plants and loads are widely spaced apart from one another, it is advisable to utilize a decentralized scheme. Local control does not require a mode of communication between other DG plants in obtaining local measurements and it is used in this research by applying the droop concept to the inverters.

\section{B. Modelling the Frequency and Voltage Droop Controllers}

Frequency is expected to change when there is a mismatch between load and the power output generated. Voltage control is also paramount to help balance the system through reactive power injection or absorption.

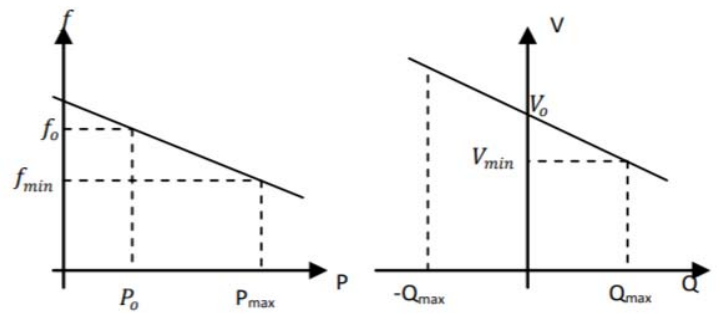

Fig. 1: $P(f), Q(V)$ conventional droop control [23]

From Fig. 1 above, the measured active $(P)$, reactive $(Q)$ power is therefore interrelated to the frequency $(f)$ and voltage (V) droop parameters of the system respectively.

$$
\begin{gathered}
f=f_{o}+K_{P f}\left(P_{o}-P\right) \\
\mathrm{V}=V_{o}+K_{Q V}\left(Q_{o}-Q\right) \\
K_{P f}=\Delta f / P_{\max } \\
K_{Q V}=\Delta \mathrm{V} / Q_{\text {max }}
\end{gathered}
$$

From equations (1) and (2) above, $f_{o}$ and $V_{o}$ are the DG plant's rated frequency and voltage; $P_{o}$ and $Q_{o}$ are the set-point reference for the inverter. The active and reactive power coefficients are $K_{P f}$ and $K_{Q V}$ which are derived from equations (3) and (4) respectively. $\Delta f$ is the maximum frequency $( \pm 2 \%$ of nominal frequency); $\Delta \mathrm{V}$ is the maximum voltage magnitude which should not exceed $\pm 10 \%$ based on EN 50160 standards [23].

\section{THE OPERATION OF GRID-FORMING INVERTERS IN AN ISLAND LOW VOLTAGE MICRO-GRID.}

In power systems, inverters can be designed to operate in either grid-forming or grid-following modes as discussed below. The grid-following inverters regulate the current and phase angle but cannot operate as standalone. Grid-forming inverters can be deployed for island microgrid and the main benefit of grid-forming inverters is that due to their voltage source characteristic they react instantaneously to grid disturbances by controlling the voltage magnitude and frequency [20]. Grid-forming mode in the inverters can be described as the DC/AC converter interaction with a non-stiff power grid or its operation in the complete absence of a power grid with synchronous generators [21]. A Grid-Forming Battery (GFB) Inverter is utilized in this research as the main generator; it is responsible for setting and synchronizing the network voltages in the island microgrid.

\section{Modelling the Low Voltage Island GRID TestBed}

For this research, the CIGRE Low Voltage (LV) benchmark [25] was modified as follows; it was disconnected from the main grid to establish an island microgrid and grid-forming 
batteries were introduced as the main generating source. The following was modelled and simulated in real time via the RTDS machine; a Wind turbine with $3 \mathrm{KVA}$ rating and two $\mathrm{PV}$ systems of 10 and 13 KVA. Controllable loads were operated using residential load curve. The PHIL test setup is shown in Fig. 2a and 2b below; the Hardware Under Test (HUT) is a 10 KVA battery energy storage system (BESS-HUT) with a $\mathrm{DC} / \mathrm{DC}$ and DC/AC converter and a controller PC (provided by Triphase) where the droop parameters for the BESS-HUT were modelled. The ancillary services provided by each DG plant and their rating is shown in Table 1 below.

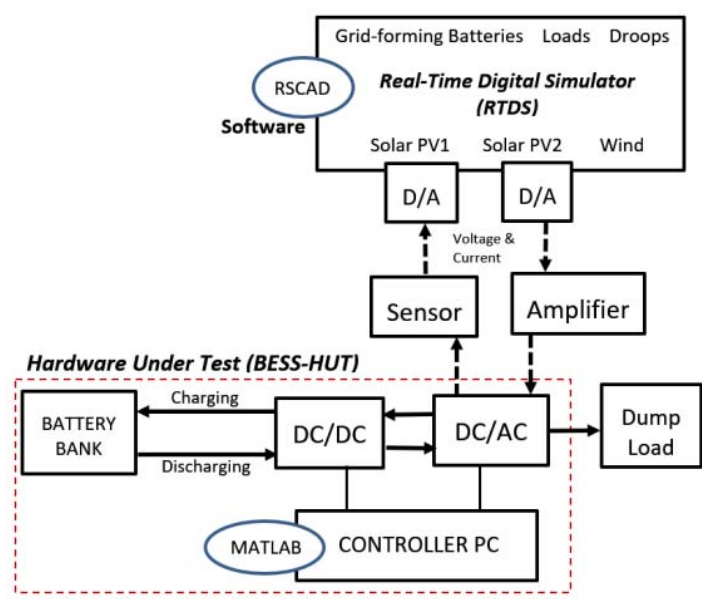

Fig. 2a: Line diagram of PHIL LV Island microgrid Test bed

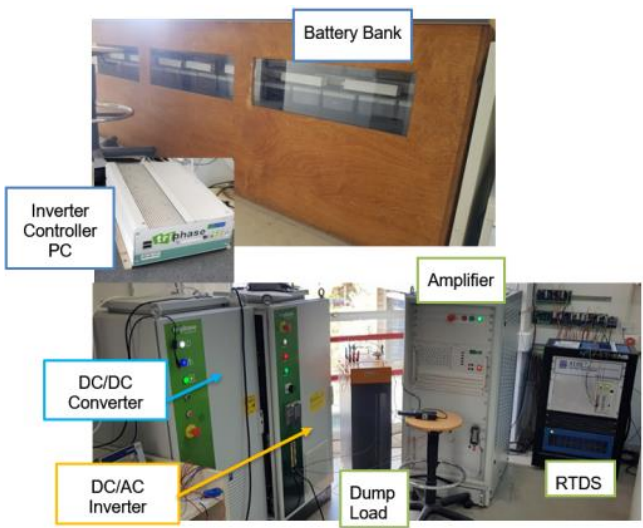

Fig 2b. PHIL Island microgrid Experimental Test Setup Facility

\begin{tabular}{|c|c|c|}
\hline DG Plants & $\begin{array}{c}\text { Rating } \\
\text { (KVA) }\end{array}$ & $\begin{array}{c}\text { Droop control modelled in } \\
\text { DG plant }\end{array}$ \\
\hline Solar PV 1 & 10 & \multirow{2}{*}{$P(f)$ and $Q(V)$} \\
\hline Solar PV 2 & 13 & \\
\hline BESS -HUT & 10 & \\
\cline { 1 - 2 } Wind & 3 & $V(V)$ only \\
\hline
\end{tabular}

TABLE 1: DG plants and the ancillary service provided via droop parameters.

The inclusion of the battery energy storage system was very significant to the test facility as the island grid required a means to store excess power from other DG plants and to dispatch power when needed as the microgrid condition changes. Five test scenarios were examined as shown in Table 2 below by combining different instances of Loads and various mix of power from the DG plants.
As is usual safety practice before any hardware device was connected in a PHIL setup, the test was first conducted using a real-time simulator. The grid-forming batteries initially served the loads in the island microgrid and the resultant profiles were examined. Furthermore, the DERs generated power and the voltage profiles were examined again; additional loads were then turned-on which triggered a reduction in the frequency of the network and the hardware battery inverter (BESS-HUT) was employed to resolve the issue. Using the $P(f)$ droop controller of the BESS-HUT modelled as a MATLAB function code, the battery inverter of the HUT then discharged the energy storage to help resolve the frequency of the island system back toward nominal value.

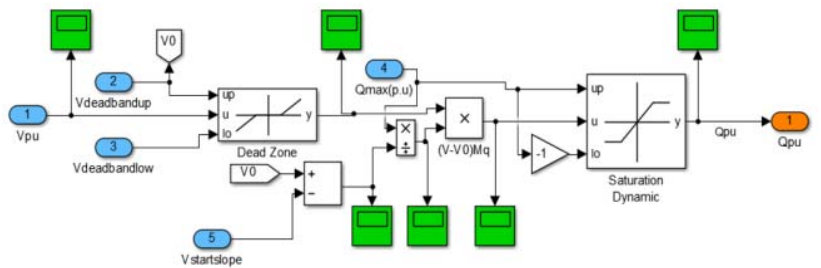

Fig. 3: $Q(V)$ droop control for Battery Inverter (HUT)

In occasions where the nominal voltage is affected due to higher inductive loads being switched on, the BESS-HUT inverter participated in reactive power compensation using the $Q(V)$ droop controller in Fig. 3 to help resolve the network voltage toward the nominal value. The BESS-HUT was charged when the situation above happens in the other way.

\begin{tabular}{|c|c|c|c|l|}
\hline $\begin{array}{c}\text { Test- } \\
\text { Case. }\end{array}$ & $\begin{array}{c}\text { Solar } \\
\mathbf{1 8 2}\end{array}$ & Wind. & Load. & \multicolumn{1}{|c|}{ Remarks } \\
\hline 1st. & L & H & M & $\begin{array}{l}\text { DERs able to meet active load } \\
\text { requirement, reactive power supplied } \\
\text { from BESS-HUT }\end{array}$ \\
\hline 2nd. & H & M & M & $\begin{array}{l}\text { BESS-HUT supports with reactive } \\
\text { power. }\end{array}$ \\
\hline 3rd. & H & H & L & $\begin{array}{l}\text { BESS-HUT charges via excess power } \\
\text { from DERs due to minimum load } \\
\text { requirement }\end{array}$ \\
\hline 4th. & L & M & H & $\begin{array}{l}\text { BESS-HUT discharges when local loads } \\
\text { are at max. to support DERs }\end{array}$ \\
\hline 5th. & X & L & H & $\begin{array}{l}\text { Evenings with high loads, GFB supply } \\
\text { active power \& DG inverters supply } \\
\text { reactive power to island grid }\end{array}$ \\
\hline L= Low, M = Medium, H = High, X = N/A or No power
\end{tabular}

TABLE 2: PHIL test scenarios with various instances of Loads and DERs

\section{RESUltS AND Discussion}

Table 2 above shows the scenarios tested in this paper; the results of the test scenarios are discussed in this section. The simulation result has been compared with the results from the PHIL test and they have similar characteristics; the PHIL test results are shown and discussed in this paper. Each test is run for about 6 seconds; the first quarter $(1.5 \mathrm{sec})$ of the result show the initial state of the PHIL testbed before application of droop controllers to the participating DG plants to correct the frequency and voltage issues experienced in the LV island microgrid. 
a) 1st Test Case-Low PV, High Wind, Medium Load

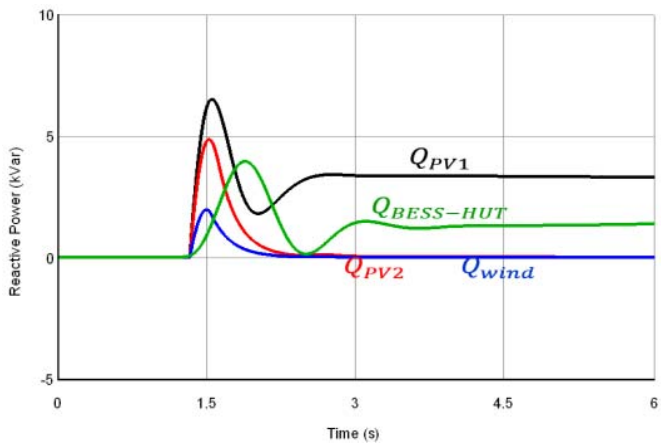

Fig. 4: Reactive Power supplied by the DG plants with droop control

In the first test case, half the load capacity was in use; the active loads were supplied by wind inverter and 25\% solar PV power generated. The Grid Forming Battery (GFB) inverter was responsible for reactive power compensation, because the droops were initially switched off as seen in Fig. 5 below. The droop controllers were then activated (at $1.5 \mathrm{sec}$ ) and there was a reduction in the reactive power that was initially supplied by the GFB inverter to local loads (from about $7.5 \mathrm{kVAr}$ to 2.5 $\mathrm{kVAr}$ ) since the PV inverter and battery hardware inverter (BESS-HUT) now supplied reactive power as shown in Fig. 4 above. As a result, the voltages were also improved in the network toward the nominal voltage value as seen in Fig. 6 (for example, L4 from $224.8 \mathrm{~V}$ to $228.8 \mathrm{~V}$ approx.).

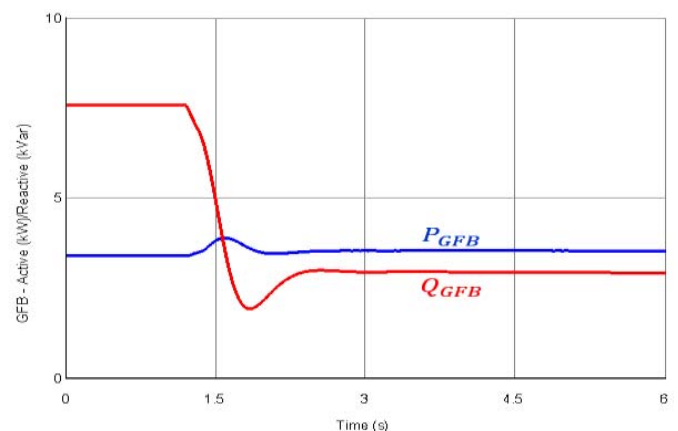

Fig. 5: Active / Reactive power from Grid-forming battery (GFB) inverter

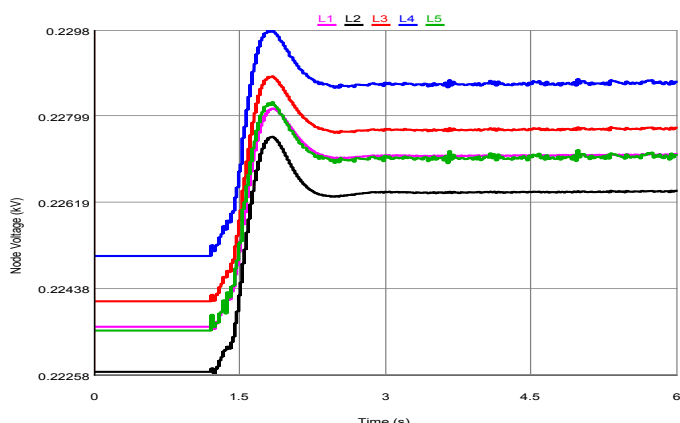

Fig. 6: Improved voltage in the Island microgrid with droop control

\section{b) 2nd Test Case-High PV, Medium Wind, Medium Load}

Due to high insolation levels in the 2nd test case, the solar PV inverters supplied maximum active power and was supported by the power generated by the wind inverter. At simulation time of 1.5 secs when the droop controllers were activated, BESS-HUT discharged to supply reactive power to the network in Fig 7 below. The activation of $P(f)$ droop controller in the PHIL test brought down the system frequency from $50.48 \mathrm{~Hz}$ to $50.28 \mathrm{~Hz}$ approx. as shown in Fig. 8 below.

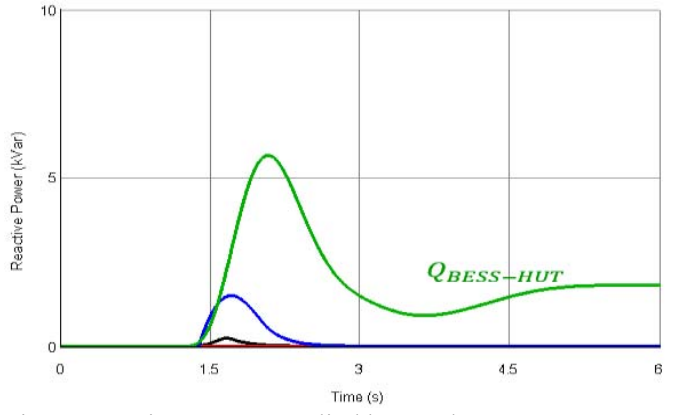

Fig. 7: Reactive Power supplied by Hardware Battery Storage (BESS-HUT) upon activating droop control

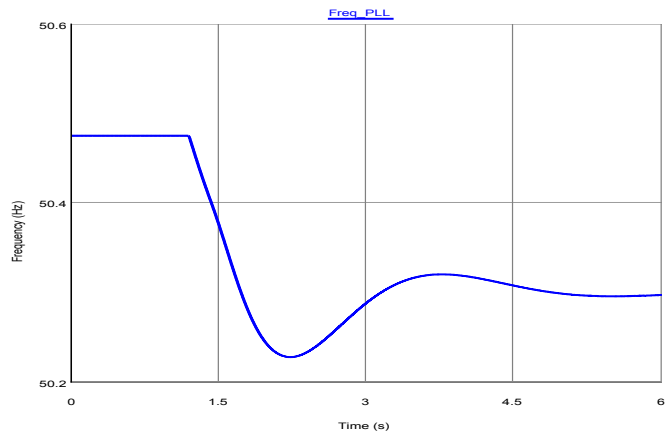

Fig. 8: Improved frequency via $P(f)$ droop in the Island microgrid

For the 3rd test case, there was a further reduction in consumption of the residential load to about $25 \%$ of the full capacity, the wind and PV inverters supplied maximum active power. As a result, the GFB inverter was able to supply reactive power to the local load. As the ratio of power generated by the DERs was higher than the load requirement, the hardware battery (BESS-HUT) were actively charged as shown in Fig. 9 upon initializing the droops. Likewise, in Fig. 9, the active power from the PV inverters were also curtailed through the droop activation for overvoltage protection.

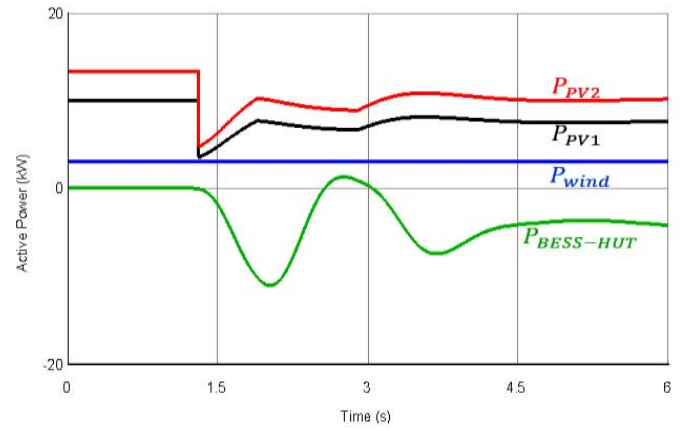

Fig. 9: Active charging of the Hardware Battery Storage (BESS-HUT) after droop activation - Active power curtailment on PV inverters via droop

The 4th test case had maximum load in operation and hence the GFB inverter initially supplied power to the loads. Upon initiating the droop controls, the PV inverters supplied reactive power with their remaining KVA rating and there was an improvement in the node voltages of the DG plants toward the nominal value. With the droop controllers triggered, the 
hardware battery BESS-HUT then discharged to support the active load requirement due to limited amount of active power from the solar PVs and the wind turbine which could be linked external factors such as weather (low insolation levels and wind speed) [34].

\section{c) 5th Test Case-No Solar PV, Low Wind, High Load}

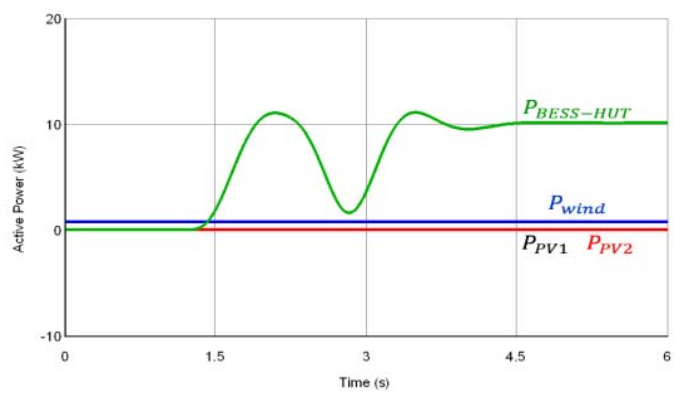

Fig. 10: Hardware Battery Energy Storage (BESS-HUT) supplying Active Power via $P(f)$ droop control

The scenario in the 5 th test case depicts evening time in a residential island network. With no solar irradiance in the evenings, the PV inverters cannot generate active power (Fig. 11). This means the inverter is available at those times and its controller is modified to generate reactive power as proposed in this research. Before activating the droop parameter, it can be seen in Fig. 12 below that the GFB inverter fed power to the Island micogrid to meet the load requirement.

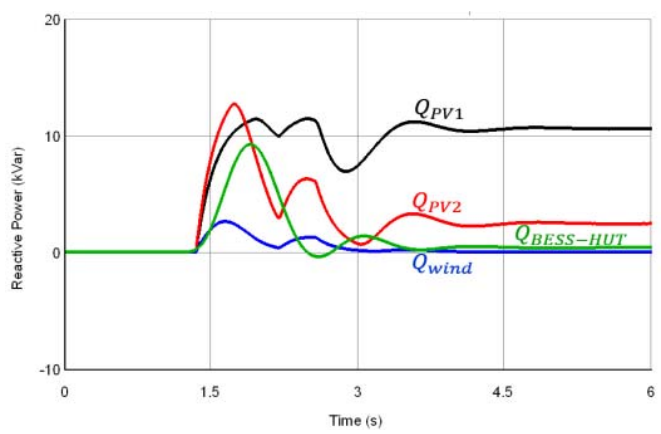

Fig. 11: Reactive Power supply from Solar PV inverters

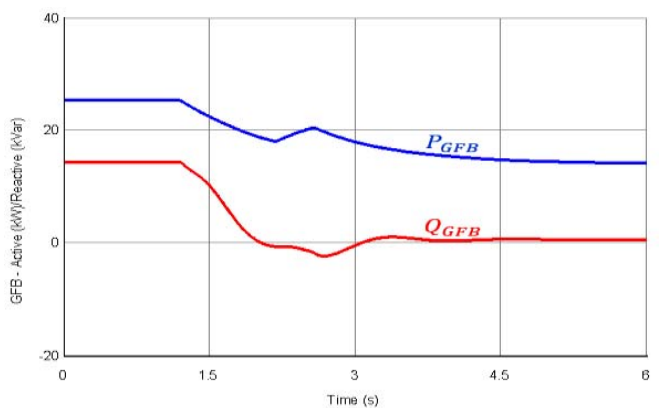

Fig. 12: Active / Reactive power from Grid-forming Battery (GFB) inverter

Due to the limited active power from the DERs to the local loads, the voltage at the nodes were affected. However, upon engaging the $P(f)$ droop, the BESS-HUT discharged active power as seen in Fig. 10 above to support the local loads. It can be seen in Fig. 13 below that the frequency in the system improved from $49.04 \mathrm{~Hz}$ to $49.5 \mathrm{~Hz}$ approx. because of the activation of the $P(f)$ droop controller; this then reduced the active power dispatched by the GFB inverter in Fig. 12. The node voltage connecting the DGs improved from about $218 \mathrm{~V}$ to $228 \mathrm{~V}$ approx. shown in Fig. 14 below when the $Q(V)$ droop controllers were activated, and the PV inverters supplied reactive power to the island network (Fig. 11). The reactive power from the GFB inverter also significantly tend toward zero because of the reactive power provision from the PV inverters.

Several operating conditions of the LV island microgrid have been examined above; it can be seen in the results presented that the application of the droop controllers did not only help maximize the functionality of the inverters but also helped improve the frequency and voltages of the overall network.

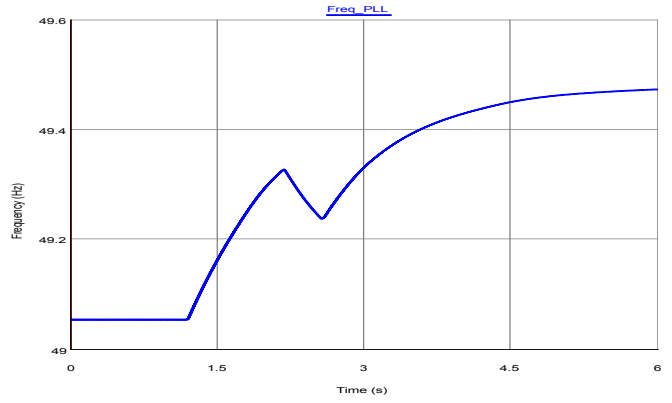

Fig. 13: Improved frequency via $P(f)$ droop in the Island microgrid

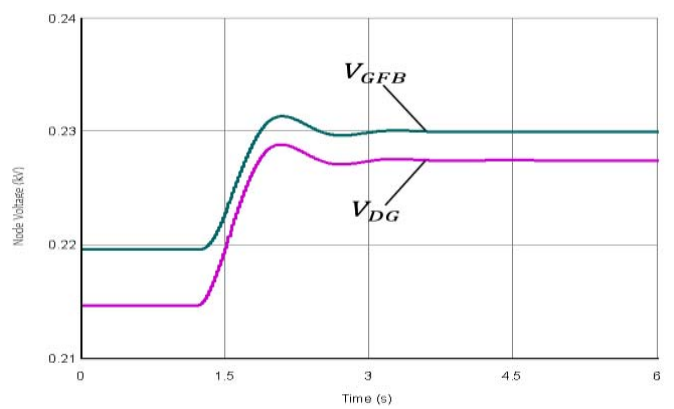

Fig. 14: Improved Node voltage in the Island microgrid with $Q(V)$ droop

\section{CONCLUSION}

The frequency and voltage issues typically experienced in LV island microgrid has been addressed in this research paper by applying droop control in the operation of the distributed generation inverters in an island system. The island system was designed as a Power Hardware in the Loop testbed (PHIL) made up of grid-forming battery, wind, and solar PV inverters modelled in a Real-Time Digital Simulator (RTDS); and a Hardware Under Test (HUT) which is a battery hardware inverter (BESS-HUT). To ensure selection of right parameters, protection of the HUT and RTDS from damage and to reinforce the performance of the overall island system, simulations were carried out initially before connecting the HUT to the RTDS for experiments. Finally, the results from the experiments above show frequency improvement in the island system via the DG inverters, active power curtailment via the PV inverters for overvoltage protection and encourages the use of droopcontrolled inverters as viable reactive power management tool. 


\section{ACKNOWLEDGMENT}

This research work has been conducted through the European Research Infrastructure (ERIGrid), funded by the European Union's Horizon 2020 Research and Innovation Programme (Grant Agreement No. 654113). The authors recognize the support of the European Research Infrastructure and appreciate its partner Smart Grids Research Unit (Smart RUE) - Electric Energy Systems (EES) research lab at ICCSNTUA Athens, Greece. The authors would like to thank Dr. Panos Kotsampopoulos and Prof. Nikos Hatziargyriou for their support.

\section{REFERENCES}

[1] Y. Zhang, J. Wang, Z. Li, Uncertainty Modelling of Distributed Energy Resources: Techniques and Challenges, Springer.com [Online], Vol 6, Iss. 2, pp 42-51

[2] T. Adefarati, R.C. Bansal, "Integration of renewable distributed generators into the distribution system: a review", IET Renewable Power Generation Journ, ISSN:1752-1416, Vol. 10, Iss. 7, pp. 873-884

[3] J.C. Vasquez et al, "Hierarchical Control of Intelligent Microgrids: Integration of Distributed Energy Resources into the Smart Grid", 2011, IEEE Industrial Electronics Magazine, Vol 4, Iss 4, pp 23 -29.

[4] G. N. Psarros, S. A. Papathanassiou, "Operation of a Wind-PV-Battery Hybrid Power Station in an Isolated Island Grid", 11th Mediterranean Conf on Power Generation, Trans., Distr. and Energy Conversion, Researchgate.net, pp 1-7

[5] H. Schaede, M. Schaede et. al, "Development of Kinetic Energy Storage Systems for Island Grids", 9th International Renewable Energy Storage Conference (IRES 2015), pp1-10

[6] T. Basso, R. DeBlasio, "IEEE Smart Grid Series of Standards IEEE 2030 (Interoperability) and IEEE 1547 (Interconnection) Status", 2012, N.R.E.L National Renewable Energy Laboratory, pp 1-9

[7] A. Eshraghi, et al, "Frequency Regulation in Island Microgrid Using Demand Response",2017, IEEE North American Power Symposium (NAPS), pp 1-6

[8] P. Kotsampopoulos et al, A Benchmark System for Hardware-in the-Loop Testing of Distributed Energy Resources, 2018, IEEE Power and Energy Tech Sys Journal, Vol 5, no.3 pp 94-103

[9] O. Tremblay, H. Fortin-Blanchette, R. Gagnon, R. Brissette, "Contribution to stability analysis of power hardware-in-the-loop simulators", 2017, IET journal on Gen, Trans \& Distr. Vol. 11, Iss. 12, pp. $3073-3079$

[10] Erik de Jong, et al "European White Book on Real-Time Power Hardware-in-the-Loop testing", 2012, pp 1-46, DERlab Report No. R005.0, ISBN: 978-3-943517-01-9

[11] M. Maniatopoulos, D. Lagos, P. Kotsampopoulos, N. Hatziargyriou, "Combined control and power hardware in-the-loop simulation for testing smart grid control algorithms", I.E.T Gen, Trans. \& Distr., 2017, Vol: 11, Iss: 12 , pp. 3009-3018

[12] K. Luo, W. Shi, H. Tang, "Implementation and value of power hardware in the loop testing bed for wind turbines integrated into grid", IET, 6th Int'l Conf on Renewable Power Generation (RPG), pp. 1635-1639

[13] S. Oyegoke, Y. Habtay, M. Maniatopoulos, P. Kotsampopoulos, S. Keates, "Power Hardware in the Loop and Ancillary Service for Voltage Regulation in Low Voltage Grid", 54th International Universities Power Engineering Conference (UPEC), Bucharest, September 2019

[14] Jason J. Poon, et al, "Hardware-in-the-Loop Testing for Electric Vehicle Drive Applications", 5th IET Int'l Conference on Power Electronics, Machines and Drives (PEMD 2010), pp 2576 - 2582

[15] P. Kotsampopoulos, et al, "The Limitations of Digital Simulation and the Advantages of PHIL Testing in Studying Distributed Generation Provision of Ancillary Services" 2015, IEEE Transaction. on Industrial. Elect, Vol. 62, No. 9, pp.5502-5515

[16] F. Huerta, M. Prodanovic, "Real-Time Power Hardware-In-The-Loop Implementation of Variable-Speed Wind Turbines", 2016, IEEE Trans. on Indus.Electr, Vol 64, Iss. 3, pp 1893-1904

[17] Y. Wang, K.T. Tan, P. L. So, "Coordinated Control of Battery Energy Storage System in a Microgrid", (2013) IEEE PES Asia-Pacific Power and Energy Engineering Conf (APPEEC), pp1-6
[18] T. L. Vandoorn et al, "Microgrids: Hierarchical Control and an Overview of the Control and Reserve Management Strategies", 2013, IEEE Industrial Electronics Magazine, Vol 7, Issue 4, pp 42 - 55

[19] X. Feng et al, "Comparison of Hierarchical Control and Distributed Control for Microgrid", 2017, Journal on Electric Power Components and Systems, Vol 45, Issue 10, pp 1043-1056

[20] G. Tembo, "Overview of the Microgrid Concept and its Hierarchical Control Architecture", 2016, Int'l Journal of Engr. Research \& Technology, Vol. 5 Issue 03, pp 50-55

[21] P. Unruh, T. Gühna, "Distributed grid-forming inverters in power grids", 7th Int'l Workshop on Integration of Solar Power into Power Systems, 2017, pp.320-326

[22] A. Tayyebi, F. Dorfler et. al, "Grid-Forming Converters - Inevitability, Control Strategies and Challenges in Future Grids Application", CIRED Workshop, 2018, Paper 0236, pp 1-5

[23] S. Manusur, M. Marei, A. Sattar, "Droop based Control Strategy for a Microgrid", 2016, Global Journal of Researches in Engineering: Elect and Electr Engr. Vol. 16 Iss. 7 Ver 1.0, pp 2249-4596

[24] S. K Sahoo, A. K Sinha, N. K Kishore, "Control Techniques in AC, DC, and Hybrid AC-DC Microgrid: A Review", 2018, IEEE Journal of Emerging and Selected topics in Power Electr, Vol. 6, no. 2, pp 738 - 759

[25] Papathanassiou. S, et.al 'A benchmark low voltage microgrid'. CIGRé Symposium. 'Power systems with Dispersed Generation: Technologies, Impacts on Development, Operation and Performances', 2005

[26] N. Ntavarinos, P. Kotsampopoulos, D. Lagos, N. Hatziargyriou, "Hardware in the Loop testing of Battery-less Hybrid Systems for Offgrid Power Supply", 13th IEEE PES Powertech, Milan, June 2019

[27] S. Oyegoke, Y. Habtay, M. Maniatopoulos, P. Kotsampopoulos, S Keates, "Dynamics of Inverter Droop Control and OLTC using Power Hardware In the Loop (PHIL)", International Conference on Energy and Sustainable Futures (ICESF) 2019, Nottingham, September 2019

[28] T. S. Ustun, H. Konish, J. Hashimoto, K. Otani, "Hardware-in-the-loop simulation-based Testing of power conditioning systems", 2018, IEEE Int'l Conf. on Indust. Electronics for Sustainable Energy Systems (IESES), pp 546-551

[29] S. Lentijo, S. D'Arco, A. Monti, "Comparing the Dynamic Performances of Power Hardware-in-the-Loop Interfaces", IEEE Transaction. on Industr. Electr, Vol. 57, No. 4, April 2010, pp1 195 - 1207

[30] K. R. Bharath, M. Krishnan Mithun, P. Kanakasabapathy, "A Review on DC Microgrid Control Techniques, Applications and Trends", 2019, Int'l Journal of Renewable Energy Research Vol.9, No.3, Sept 2019, pp 1-11

[31] R. Jackson et al., "A Self-Frequency Restoration Control Based on Droop Strategy for Autonomous Microgrid", 2019, Int'l Journal of Renewable Energy Research, Vol.9, No.2, June 2019, pp 749 -756

[32] J. Kumara, A. Agarwalb, V. Agarwala "A review on overall control of DC microgrids" [Online] Elsevier via sciencedirect.com, 2019, Jour. of Energy Storage, Vol. 21, pp113-138

[33] W. Cao et al., "Improved Droop Control Method in Microgrid and Its Small Signal Stability Analysis," 2014, 3rd Int'l Conf. on Renewable Energy Research and Applications, pp.197-202

[34] A. Abdulkarim et al., "Effect of Weather and the Hybrid Energy Storage on the Availability of Standalone Microgrid", 2016, Int'l Jour. of Renewable Energy Research, Vol.6, No.1, pp189 -198 